\title{
In Situ HAADF-STEM Imaging and Tomography of AuIr Bimetallic Catalysts
}

\author{
Chang Wan $\operatorname{Han}^{1}$, Ernesto E. Marinero ${ }^{1}$, Antonio Aguilar ${ }^{2}$, Rodolfo Zanella ${ }^{2}$, and Volkan Ortalan ${ }^{1}$ \\ 1. School of Materials Engineering, Purdue University, West Lafayette, IN, USA \\ 2. Centro de Ciencias Aplicadas y Desarrollo Technologico, Universidad Nacional Autonoma de \\ Mexico, Mexico City, Mexico
}

High-angle annular dark field (HAADF) STEM is an indispensable technique for analyzing heterogeneous catalysts, in particular those comprising high atomic number $(Z)$ metallic nanoparticles (NPs) dispersed on low-Z supports. Readily interpretable atomic-scale Z-contrast imaging with high spatial resolution spectroscopy, including X-ray energy dispersive spectroscopy (EDS) and electron energy loss spectroscopy (EELS), allows researchers to investigate structural information such as dimensions, morphologies, and size distribution of catalytic NPs as well as their material chemistry (e.g., chemical composition, bonding of catalytic particles with supports at interface, etc.).

Although HAADF-STEM has been very successfully utilized in the field of heterogeneous catalysis, it leaves room for improvement when applied to in situ environmental studies, which is crucial to understand the dynamic processes occurring during a catalytic reaction. It has been shown that the morphology of supported NPs dynamically changes with the variation of the ambient gas composition to which the NPs are exposed [1,2]. Therefore, to obtain a fundamental understanding in catalytic systems concerning the elucidation of the reaction mechanisms, the identity of the catalytically active species and the mechanisms of deactivation, it is imperative to investigate catalytic systems in the operando conditions. Recently developed environmental TEM (ETEM) opens up many possibilities to investigate dynamical processes in catalysts in real time, such as fluxionality, surface diffusion/sintering of nanoparticles, and morphological changes under reactive conditions, etc. Besides HAADF-STEM imaging, electron tomography utilizing HAADF-STEM has proven to be vital in determining complete 3D size, shape, and distribution of dispersed nanostructures with a volumetric resolution of $1 \mathrm{~nm}$ [3]. By combining HAADF-STEM tomography with ETEM capability, dynamic behaviors of catalysts can be more thoroughly investigated. As a demonstration of the power of in situ HAADF-STEM imaging and tomography, our investigation on AuIr bimetallic catalysts will be presented.

It has been demonstrated that the addition of $\mathrm{Ir}$ to $\mathrm{Au}$ is a promising route to solve the problem of instability against sintering of Au catalysts [4]. Previously, the origin of enhanced stability of AuIr bimetallic catalysts has been attributed to the formation of $\mathrm{IrO}_{2}$ between $\mathrm{Au}$ and the $\mathrm{TiO}_{2}$ support [5]. In our work, we have observed enhanced stability in AuIr bimetallic catalysts without the formation of the $\mathrm{IrO}_{2}$ phase. Figure 1 shows HAADF-STEM images of (a) $\mathrm{AuIr} / \mathrm{TiO}_{2}$ and (b) $\mathrm{Au} / \mathrm{TiO}_{2}$ after hydrogen heat treatment at $500^{\circ} \mathrm{C}$ for $2 \mathrm{hrs}$. Although $\mathrm{IrO}_{2}$ is absent in the $\mathrm{AuIr} / \mathrm{TiO}_{2}$ sample, smaller average size $(2.44 \pm 0.71 \mathrm{~nm})$ and more uniform distribution are evident than for the $\mathrm{Au} / \mathrm{TiO}_{2}$ sample. The latter exhibits an average size of $5.71 \pm 3.71 \mathrm{~nm}$ and non-uniformity in the distribution of Au particles. This is suggestive that other mechanisms are responsible for the enhanced stability observed in AuIr bimetallic catalysts. Moreover, AuIr/TiO 2 bimetallic catalysts show higher catalytic activity in $\mathrm{CO}$ conversion compared to $\mathrm{Au} / \mathrm{TiO}_{2}$ [Figure 1. (c)]. The higher catalytic activity of AuIr bimetallic catalysts over pure $\mathrm{Au}$ catalysts can be attributed to the smaller particle size and therefore a higher density of active sites present in the AuIr. 
Despite the importance of AuIr bimetallic catalysts, a detailed atomic-level picture for the sintering suppression effect of $\mathrm{Ir}$ in $\mathrm{Au} / \mathrm{TiO}_{2}$ is not yet available. Likewise, the relationship between catalytic activation parameters (temp, gas ambient) and the resulting microstructure and the mobility of AuIr particles on support has also not been investigated. We utilize in situ HAADF-STEM imaging and tomography [Figure 2] to reveal the physics behind the sintering stability and higher catalytic activity of $\mathrm{AuIr} / \mathrm{TiO}_{2}$. Catalysts samples are intermittently exposed to the reaction conditions $\left(\mathrm{H}_{2}\right.$ ambient at $500^{\circ} \mathrm{C}$ or CO ambient at room temp.) in the environmental sample stage of the FEI Titan 80-300 ETEM system. The tilt series for HAADF-STEM tomography are acquired under vacuum conditions at room temperature during which no catalytic reactions occur. Obtained results showing catalytic NPs' 3D size and spatial distribution evolution under reaction conditions with time will be presented.

\section{References}

[1] Hansen, P. L. et al, Science, 295 (2002), p. 2053-2055.

[2] Yoshida, H. et al, Science, 335 (2012), p. 317-319.

[3] Arslan, I. et al, Science, 309 (2005), p. 2195-2198.

[4] Liu, Z. P. et al, Physical Review Letters, 93 (2004), p. 151602-1

[5] Gomez-Cortes, A. et al, The Journal of Physical Chemistry C, 113 (2009), p. 9710-9720.

[6] RZ and AA acknowledge financial support given by projects CONACYT 130407 and

PAPIIT-UNAM 103513.

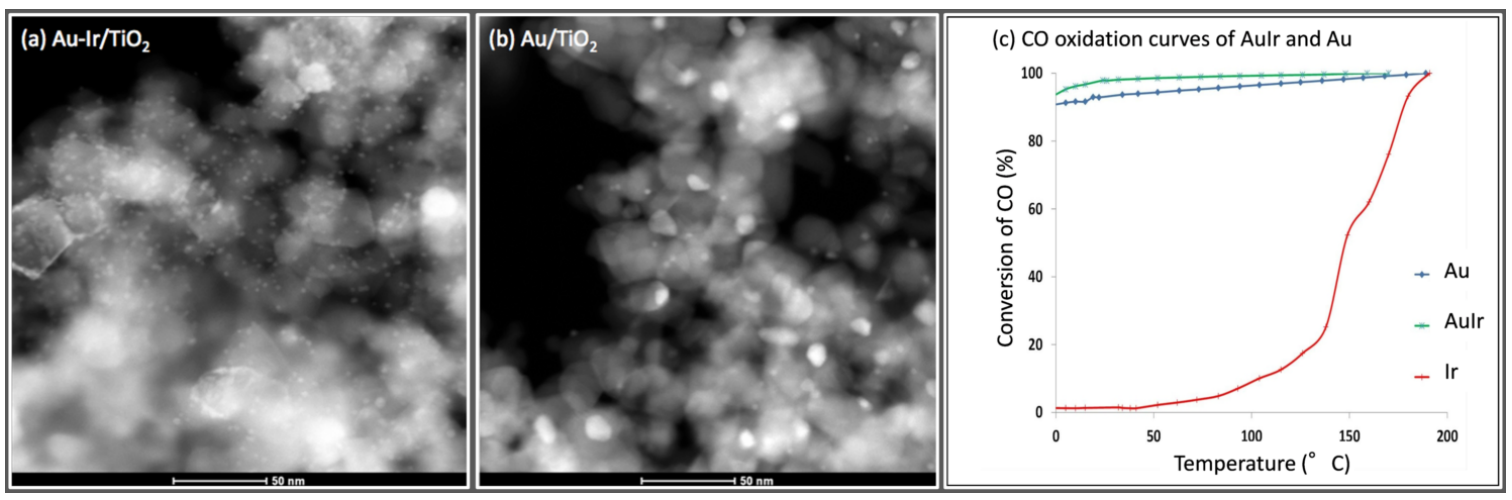

Figure 1. HAADF-STEM images of (a) $\mathrm{AuIr} / \mathrm{TiO}_{2}$, (b) $\mathrm{Au} / \mathrm{TiO}_{2}$, and $\mathrm{CO}$ oxidation curves of $\mathrm{AuIr} / \mathrm{TiO}_{2}$ and $\mathrm{Au} / \mathrm{TiO}_{2}$ (c) as a function of temperature

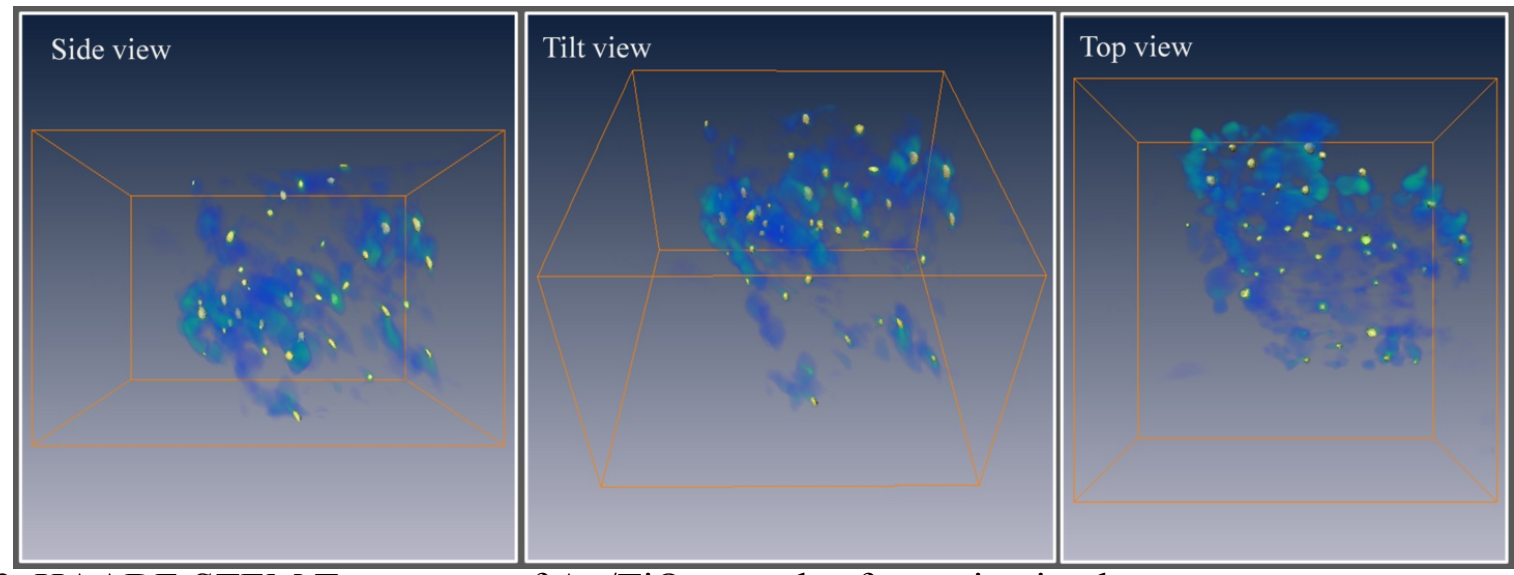

Figure 2. HAADF-STEM Tomogram of $\mathrm{Au} / \mathrm{TiO}_{2}$ sample after activation heat treatment 